\title{
BMJ Open Quality Better patient safety: implementing exploration and exploitation learning in daily medical practice
}

\author{
Ayala Kobo Greenhut, ${ }^{1}$ Racheli Magnezi, ${ }^{1}$ Izhar Ben Shlomo ${ }^{2,3}$
}

To cite: Kobo Greenhut A, Magnezi R, Ben Shlomo I. Better patient safety: implementing exploration and exploitation learning in daily medical practice.BMJ Open Quality 2017;6:e000015. doi:10.1136/ bmjoq-2017-000015

Received 26 January 2017 Revised 20 July 2017 Accepted 21 July 2017

\section{CrossMark}

${ }^{1}$ Department of Management, Bar-llan University, Ramat Gan, Israel

${ }^{2}$ Assisted Reproduction Unit, Department of Obstetrics and Gynecology, Baruch Padeh Medical Center, Tiberias, Israel

${ }^{3}$ Faculty of Medicine in the Galilee, Bar-Ilan University, Safed, Israel

Correspondence to Professor Izhar Ben Shlomo; ibenshlomo@poria.heath.gov.il

\section{ABSTRACT}

Background Current medical daily practice relies on guidelines, protocols and procedures (GPPs), which require exploitation. However, diagnosis, treatment, risk management and process improvements require exploration. Physician are often unable to switch between exploitation and exploration. This study tested a new approach to facilitate switching that included (1) a new 'thinking protocol' that encouraged leaping from exploitation to exploration and (2) a GPP that encouraged leaping from exploration to exploitation.

Method Two hundred students were divided into four groups. The groups received a set of tasks that required switching between exploitation and exploration. Three groups received the thinking protocol, the GPP, or both, and the fourth group served as control.

Results With each additional task, all groups increased exploitative tendency $(p<0.0001)$. The two groups with the thinking protocol leaped from exploitation to exploration $(p<0.0001)$; the other two groups remained in exploitation $(p=0.1173, p=0.0758)$. For the groups that employed exploration learning, the group that received the GPP switched back to exploitation $(p<0.0001)$, but the other group remained in exploration ( $p=0.2363)$.

Conclusion Despite the importance of timely leaping between exploration and exploitation, in some events, medical teams fail to make the appropriate leap. We suggest to use our novel approach and to encourage the leaping between exploration and exploitation in daily medical practice, to enable the prevention of medical errors and to enhance the effectiveness of risk managements and process improvements.

\section{INTRODUCTION}

The primary purpose of this study was to develop a new approach to encourage leaping between exploration and exploitation learning in medical practice, which we predicted will improve patient safety in medical practice. Specifically, our model was designed to encourage both adherence to predetermined written guidelines, protocols and structured procedures (GPPs), when required, and 'leaping' from one concept, diagnosis or treatment plan to another, when required.

Current medical practice encourages adherence to GPPs. GPPs are based on current evidence and experience; they provide a sense of security, organisation and order. In most instances, adhering to GPPs results in optimal patient care. GPP adherence also provides peace of mind to the caregiving team. Nevertheless, there are cases where thinking 'outside the box' is frequently required. For instance, thinking outside the box may be required in complex situations, when it is not clear what GPP would be best suited to a specific patient, or when replacing the chosen GPP with an alternative (leaping from one diagnosis or treatment plan to another). Risk management methods, such as FMEA (failure mode and effect analysis, and processes improvements concepts, such as PDCA (plan-do-study-act cycle), are based on identifying risks and opportunities for improvements; ${ }^{1}$ the ability to think 'outside the box' during risk management and process improvements is important.

Working according to GPPs is a form of exploitation learning. It emphasises standardisation, uniformity, low risk, consistency and low variance. In contrast, searching for a new diagnosis, new treatment plan, risks and opportunities for improvement is a form of exploration learning. It emphasises thinking outside the box and taking discretionary measures; it is associated with high variability and high risks. Exploration learning is essentially the approach used in research and development environments.

There is no doubt about the benefits and importance of both exploration and exploitation learning in medical care. However, both medical errors and failure to identify risks and opportunities for improvements frequently result from a physician's inability to simultaneously accelerate both of them, or rather smoothly switch between the two. ${ }^{23}$ This inability to switch behaviour lies in the variation-reducing nature of exploitation learning. Exploitation learning restricts the development of alternatives, ${ }^{4}$ hampers the discretion of employees ${ }^{5}$ and leads to resistance to change and momentum; thus, 


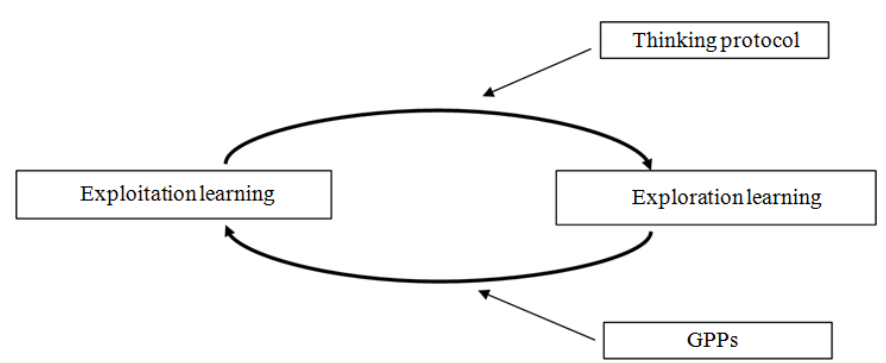

Figure 1 The leaping model. GPP, guidelines, protocols and procedures.

exploitation learning inhibits variability ${ }^{4}$ by promoting the 'easy route' of relying on habitual routines. Moreover, well learnt guidelines and procedures generally contribute to the comfort that an individual member feels within the department. When GPPs are well learnt, the confidence in an individual's role in the department increases; the risk of 'doing the wrong thing' decreases; and the likelihood of being perceived as deviant is reduced. As a result, GPPs entrap physicians into remaining within common and preselected GPPs (closed loop); in contrast, exploration learning requires leaping to alternative approaches.

Two approaches have been introduced to explain how to achieve both exploration and exploitation learning. The first approach, known as 'ambidextrous', refers to highly differentiated, but weakly integrated subunits. ${ }^{6}$ According to this approach, organisational units should specialise in either exploration or exploitation learning. Exploration and exploitation units should be located in different places, have different personnel and even be related to different suborganisations. ${ }^{4}$ In medicine, this translates into one group of teams that function according to GPPs, and another group of teams that 'think outside the box'. The second approach, known as 'punctuated equilibrium', refers to a temporal, rather than organisational, structure differentiation. It suggests that organisational transformations can be achieved by cycling through long periods of exploitation learning and short bursts of exploration learning. ${ }^{7}$ This approach enables organisations to balance between exploration and exploitation learning, as they shift from one activity to another. ${ }^{8}$

Obviously, neither of these approaches can be implemented in medicine. The ambidextrous approach is impractical for medical practice, because the same people need to comply with GPPs and think outside the box, when necessary. The punctuated equilibrium approach is difficult to apply to medical practice, because the need to shift between GPPs and thinking outside the box is unpredictable; it depends on situations, rather than time.

To encourage leaping from exploitation to exploration learning, variability in individual beliefs should be encouraged. ${ }^{9}$ Interdisciplinary and external teams are known to create such variability. ${ }^{10-13}$ Multidisciplinary teamwork identifies risks and opportunities too frequently, only partially. ${ }^{14}$ Their work is essential for the delivery of high quality and safe patient care,${ }^{15}$ and the use of both interdisciplinary and external teams is time-consuming, expensive and difficult to manage. ${ }^{12}{ }^{16}$ Moreover, according to our leaping model, the need for leaping occurs frequently, and interdisciplinary and external teams cannot be readily available in a routine manner. As a result, daily medical care requires alternative, lean elements that encourage leaping from exploitation to exploration learning.

In this study, we describe a 'Leaping model', which enabled the coexistence of exploration and exploitation learning in medical practice. To validate our model, we tested the following hypotheses in a classroom setting, before taking our model into real medical practice settings:

- Routine work encourages exploitation learning.

- A 'thinking protocol' can lead to leaping from exploitation to exploration learning.

- Working according to GPPs can induce leaping from exploration to exploitation learning.

\section{METHODS}

\section{The leaping model}

Our leaping model (figure 1) illustrates how, in medical practice, teams leap from one mode of learning to another-back and forth-over time. We created the thinking protocol as a lean, readily applicable instrument, that encouraged leaping from exploitation to exploration learning. The thinking protocol comprised a set of questions known to evoke an explorative approach. ${ }^{9} 10$ The thinking protocol encouraged discernment by teaching individuals to perceive themselves as having several choices of how to perform their tasks. ${ }^{10}$ Figure 2 shows a flow chart of the thinking protocol for medical care. The thinking protocol was implemented as a printed form (figure 3). A completed form, pertaining to the cardiological department in Poria Hospital is shown in figure 4.

GPPs control the variance in processes and outcomes ${ }^{17}$ and they reduce the variance and ambiguity ${ }^{18}$ in decisions made by personnel. Thus, we also created a set of GPPs to encourage a drift from exploration to exploitation. The GPPs comprised a set of instructions (checklist) that encouraged exploitation.

\section{Model implementation}

The participants of the study were quality engineering students. Our method tested (1) whether routine work encouraged exploitative; (2) whether the thinking protocol encouraged leaping from exploitation to exploration and (3) whether a checklist (GPP) could induce a transition from exploration to exploitation. This method was implemented with students. The students were divided into four groups (A-D). In each group, the students were assigned to pairs, where one member was the participant and the other was the observer. The participants were asked to 'think aloud' to enable the observers to complete the questionnaire. The participants received eight instructions that were given one at a time; and they were carried out with Lego building blocks. The instructions were: 


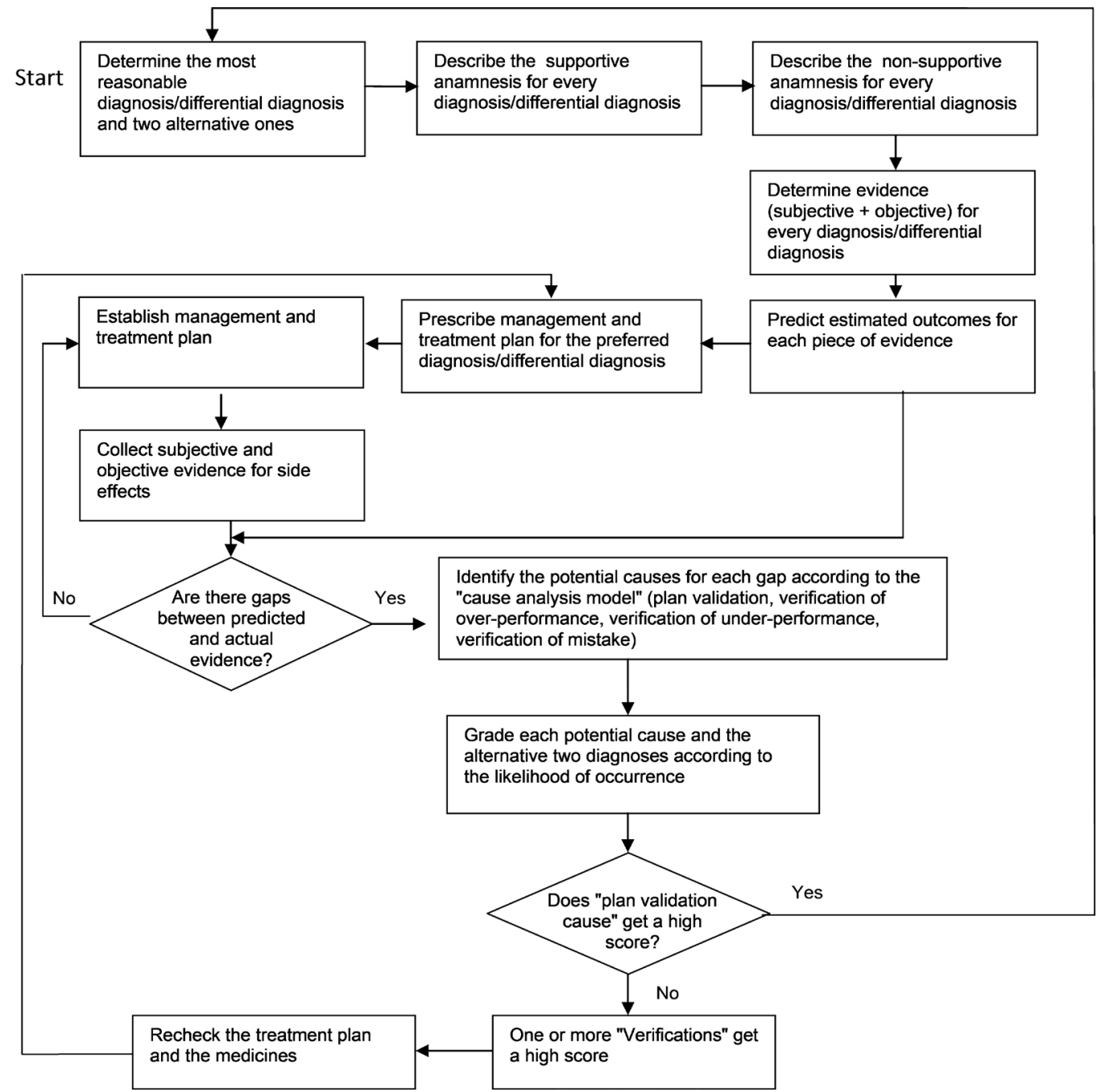

Figure 2 Flow chart of the thinking protocol.

- Create a reasonable shelter for a Lego doll that, in real dimensions, could be used for humans.

- Give a name to the shelter.

- The shelter should have doors.

- The shelter should have windows.

- The shelter should have a radio.

- The shelter should have seats.

- Modify the shelter in such a way that it can move to the next room.

- Modify the shelter in such a way that it can float on water.

Group A received the instructions without interruption (control). Group B received the instructions, and on receiving instruction 7 , they received the thinking protocol. Group $\mathrm{C}$ received the instructions, and on receiving instruction 8 , they received the GPP checklist. Group D received the instructions, and on receiving instructions 7 and 8, they received the thinking protocol and the GPP checklist, respectively.

The thinking protocol is a questionnaire that allows the participant to determine whether a gap occurred between the current design and each of the requirements. When the participant recognised a gap, he was asked to estimate whether the gap occurred due to: (1) a wrong concept, (2) the wrong implementation of a good concept, (3) overdoing a step in the implementation (adding unnecessary elements) of a good concept, or (4) missing a step in the implementation (deleting necessary elements) of a good concept. When the most likely cause for the gap was a wrong concept, the participant was asked to consider alternative concepts. Otherwise, the participant was instructed to check the implementation of the concept.

The GPP checklist comprised a list of five instructions for completing the task, as follows: (1) read the requirement and determine its influence on the design; (2) plan the implementation; (3) obtain the required Lego blocks; (4) implement your plan; (5) check whether the plan fulfils the requirement.

The observer was asked to rate the participant's response to each instruction, based on a scale from 1 ( $t o$ a slight extent) to 3 (to a large extent). Exploration instructions were assessed by rating the following responses: 'a lot of thought and discretion was needed'; 'new concepts or principles were needed'; 'new skills that were not 


\begin{tabular}{|c|c|c|c|c|c|}
\hline \multicolumn{6}{|l|}{ Background: } \\
\hline & & & & \multicolumn{2}{|c|}{$\begin{array}{c}\text { Most reasonable diagnosis/differential } \\
\text { diagnosis }\end{array}$} \\
\hline \multicolumn{4}{|c|}{ Diagnosis/differential diagnosis Description } & & \\
\hline \multicolumn{4}{|c|}{ Supportive history } & & \\
\hline \multicolumn{4}{|c|}{ Non-supportive history } & & \\
\hline \multirow{4}{*}{$\begin{array}{l}\text { Subjective } \\
\text { evidence }\end{array}$} & \multicolumn{3}{|c|}{ Subjective evidence description } & & \\
\hline & \multicolumn{3}{|c|}{ Predicted results } & & \\
\hline & \multicolumn{3}{|c|}{ Actual results } & & \\
\hline & \multicolumn{3}{|c|}{$\begin{array}{l}\text { Occurrence of gap between actual and predicted } \\
\text { results (+/-) }\end{array}$} & & \\
\hline \multirow{4}{*}{$\begin{array}{l}\text { Objective } \\
\text { evidence }\end{array}$} & \multicolumn{3}{|c|}{ Objective evidence description } & & \\
\hline & \multicolumn{3}{|c|}{ Predicted results } & & \\
\hline & \multicolumn{3}{|c|}{ Actual results } & & \\
\hline & \multicolumn{3}{|c|}{$\begin{array}{l}\text { Occurrence of gap between predicted and actual } \\
\qquad \text { outcomes }(+/-)\end{array}$} & & \\
\hline \multicolumn{6}{|c|}{ In case of a gap between predicted and actual results } \\
\hline & & $\begin{array}{c}\text { Cause } \\
\text { description }\end{array}$ & $\begin{array}{l}\text { Estimate the } \\
\text { that each ca } \\
\text { produce the } \\
\text { gap ( } 1\end{array}$ & $\begin{array}{l}\text { ikelihood } \\
\text { se could } \\
\text { specific } \\
10)\end{array}$ & $\begin{array}{l}\text { Recommendation when it is } \\
\text { highly likely that the cause } \\
\text { produced the specific gap }\end{array}$ \\
\hline \multirow{5}{*}{$\begin{array}{l}\text { Related } \\
\text { causes }\end{array}$} & Mistake & & & & \multirow{3}{*}{$\begin{array}{l}\text { Recheck the treatment plan } \\
\text { implementation }\end{array}$} \\
\hline & Over-performance & & & & \\
\hline & Under-performance & & & & \\
\hline & Diagnostic error & & & & $\begin{array}{l}\text { Determine alternative } \\
\text { diagnosis }\end{array}$ \\
\hline & $\begin{array}{l}\text { Treatment plan } \\
\text { concept error }\end{array}$ & & & & $\begin{array}{l}\text { Determine alternative } \\
\text { treatment plan }\end{array}$ \\
\hline
\end{tabular}

Figure 3 Thinking protocol form.

needed in the previous tasks were needed here'; 'significant changes were made'; and 'searching for new possibilities was carried out'. Exploitation instructions were assessed by rating the following responses: 'concepts or principles that were used in previous tasks were required here'; 'compared to previous tasks, minor changes were carried out'; 'activities were based on experience acquired during previous tasks'; 'activities were based on knowledge acquired during previous tasks'; and 'was it clear to you (the participant) how to conduct the activities?'.

The independent variables were the requirements (the eight instructions). The dependent variables (outcomes) were the score for exploration and the score for exploitation.

\section{RESULTS}

Data were collected for 25 pairs of students in each of the four groups (groups A to D, 50 students in each group). The study included 200 participants. The results are shown in figure 5.

When receiving the first instruction, the condition of all groups was characterised as low exploitation. As they progressed through the instructions, all groups exhibited 


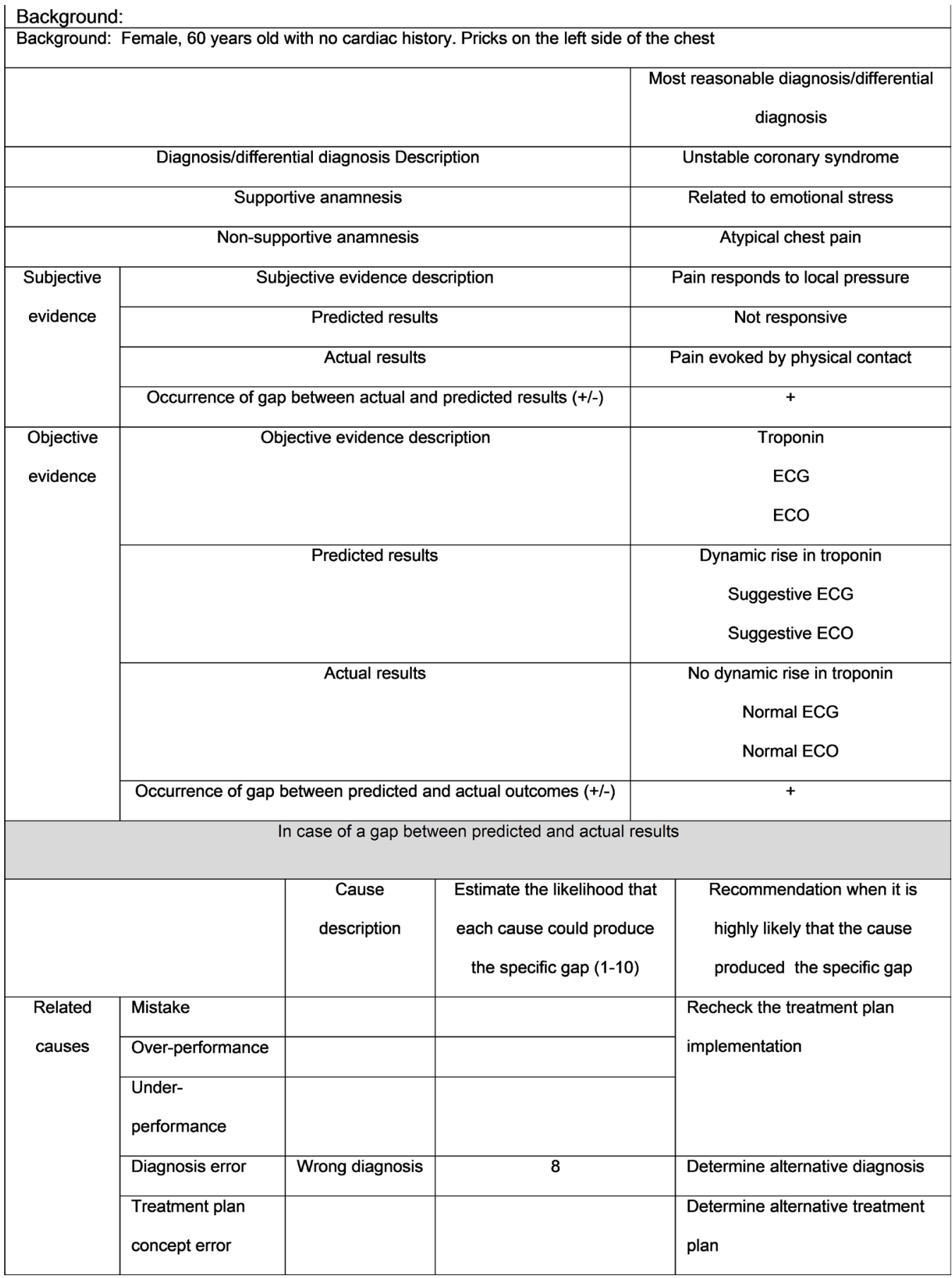

Figure 4 Detailed example of thinking protocol form. Alternative diagnosis: whole chest pain. ECO, echocardiography.

increasingly more exploitation $(\mathrm{p}<0.0001)$. Instruction 7 required leaping from exploitation to exploration, because after completing the first six instructions to build a house or a bridge, the seventh instruction required the participant to change the initial concept from a house or bridge to some kind of vehicle. Immediately before receiving instruction 7 , the condition of all groups was characterised as high exploitation. At this point, two groups received the thinking protocol and two did not. Both groups that implemented the thinking protocol leaped from exploitation to exploration $(\mathrm{p}<0.0001)$. The other two groups continued with exploitation $(\mathrm{p}=0.1173$, $\mathrm{p}=0.0758)$. Instruction 8 required leaping from exploration to exploitation; because no innovative idea was required (Lego blocks float in water). At this point, two groups had been exhibiting exploitation and two groups had been exhibiting exploration. Both groups that had exhibited exploitation continued with exploitation learning, whether they received or did not receive the checklist $(\mathrm{p}=0.0385, \mathrm{p}=0.0181$, respectively). However, the two groups that had exhibited exploration responded differently to instruction 8 . The group that received the checklist exhibited leaping to exploitation $(p<0.0001)$, and the group that did not receive the checklist continued 


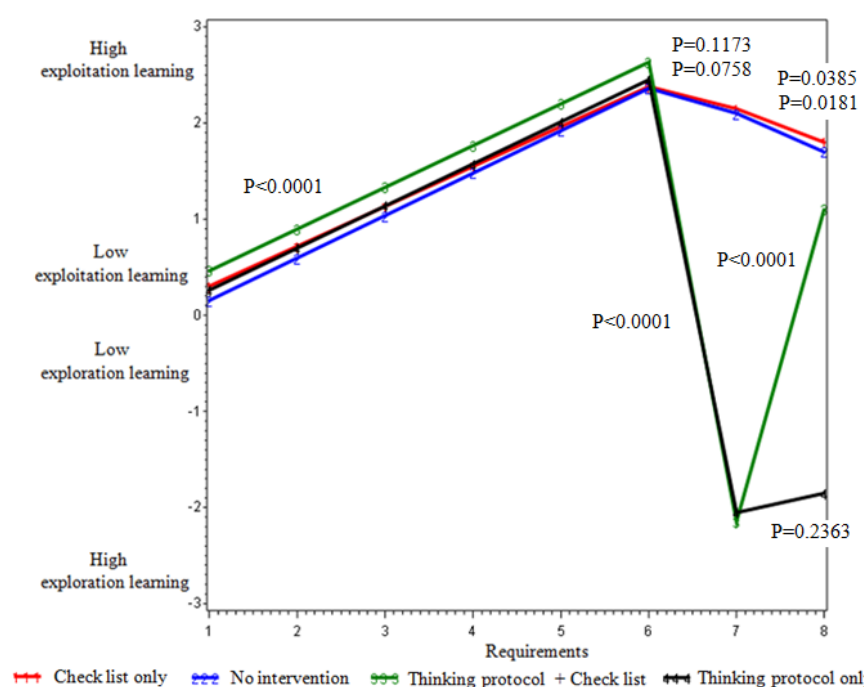

Figure 5 Test results.

with exploration $(p=0.2363)$. The participants of this group began to look for innovative concepts, such as a submarine.

\section{CONCLUSIONS}

Leaping from exploitation to exploration at appropriate times is important in preventing intellectual fixation in a situation that requires consideration, creativity and risk-taking. Leaping from exploration to exploitation at appropriate times is important in preventing undesirable situations, such as wasting resources and missing opportunities to prevent the deterioration of a given situation. Moreover, in medical practice, leaping from exploration to exploitation at inappropriate times can also lead to deterioration in a patient's condition. When the patient's state becomes unstable, the clinician must adhere to a predetermined set of actions (exploitation) taken in an attempt to stabilise the patient's condition.

Despite the importance of timely leaping between exploration and exploitation, in some events, medical teams fail to make the appropriate leap. ${ }^{2}$ Indeed, our results showed that routine work encouraged high exploitation. Within exploitation, it was difficult to recognise the need to leap into exploration. However, we found that when the thinking protocol was implemented, individuals could recognise the need for exploration, and new concepts were established. The thinking protocol was critical in preventing unreasonable adherence to a single diagnosis or treatment. Conversely, within exploration, it was difficult to recognise the need to leap into exploitation. However, we found that, when the GPP checklist was implemented, individuals could recognise the need for exploitation; thus, they stopped searching for new concepts, and began working according to a protocol. Both the GPP checklist and thinking protocols are sustainable. As we have seen in this research, both can be implemented identically by different teams.

Our leaping model combined the two accepted, but contradictory, approaches for establishing a balance between exploration and exploitation. On one hand, consistent with the ambidextrous approach, we posited that exploitation and exploration were separate states, and that leaping between them required conscious effort. On the other hand, consistent with the punctuated equilibrium approach, because leaping between is dynamic and generally requires short durations, we posited that the two also existed simultaneously. Moreover, although several studies have discussed the existence of leaping, ${ }^{9}{ }^{19-21}$ most authors have suggested that leaping occurs over a relatively long period of time. On the other hand, we suggest that leaping can occur over very short time periods, like days, or even hours.

In the future our insights will be investigated during the implementation of our model (leap from exploitation to exploration) in daily medical practice multidisciplinary teamwork including patients and caregivers in different areas as risk management and process improvement.

Contributors AKG and IBS planned the study design, and AKG performed the tests. All authors analysed the results and participated in the drafting and completion of the manuscript.

Competing interests None declared.

Provenance and peer review Not commissioned; externally peer reviewed.

Open Access This is an Open Access article distributed in accordance with the Creative Commons Attribution Non Commercial (CC BY-NC 4.0) license, which permits others to distribute, remix, adapt, build upon this work non-commercially, and license their derivative works on different terms, provided the original work is properly cited and the use is non-commercial. See: http://creativecommons.org/ licenses/by-nc/4.0/

(c) Published by the BMJ Publishing Group Limited. For permission to use (where not already granted under a licence) please go to http://www.bmj.com/company/ products-services/rights-and-licensing/

\section{REFERENCES}

1. Taylor MJ, McNicholas C, Nicolay C, et al. Systematic review of the application of the plan-do-study-act method to improve quality in healthcare. BMJ Qual Saf 2014;23:290-8.

2. Kobo-Greenhut A, Notea A, Ruach M, et al. Time to follow guidelines, protocols, and structured procedures in medical care and time to leap out. Risk Manag Healthc Policy 2014;7:233-7.

3. Greenhut AK, Magnezi R, Ben-Shlomo I. Does the implementation of quality standards freeze action modes? Total Quality Management \& Business Excellence 2016;1:1-8.

4. Benner MJ, Tushman ML. Exploitation, exploration, and process management: the productivity dilemma revisited. Academy of Management Review 2003;28:238-56.

5. Cropanzano R, Byrne ZS, Bobocel DR, et al. Moral virtues, Fairness Heuristics, Social Entities, and other denizens of organizational Justice. J Vocat Behav 2001;58:164-209.

6. Ghemawat P, Ricart Costa JEI, Costa JR. The organizational tension between static and dynamic efficiency. Strategic Management Journal 1993;14:59-73.

7. Gupta AK, Smith KG, Shalley CE. The leaping between exploration and exploitation. Academy of Management Journal 2006;4:693-706.

8. Romanelli E, Tushman ML. Organizational transformation as punctuated equilibrium: an empirical test. Acad Manage $J$ 1994;37:1141-66.

9. March JG. Exploration and exploitation in Organizational Learning. Organization Science 1991;2:71-87.

10. Amabile TM, Conti $\mathrm{R}$, Coon $\mathrm{H}$, et al. Assessing the work environment for creativity. Acad Manage J 1996;39:1154-84.

11. Meyer AN, Singh H, Graber ML. Evaluation of outcomes from a national patient-initiated second-opinion program. Am J Med 2015;128:e25-e33. 
12. Gardner G, Gardner A, O'Connell J. Using the Donabedian framework to examine the quality and safety of nursing service innovation. J Clin Nurs 2014;23:145-55.

13. Taylor A, Helfat CE. Organizational linkages for surviving Technological Change: complementary assets, Middle Management, and ambidexterity. Organization Science 2009;20:718-39.

14. Kobo-Greenhut A, Megnezi R, Reuveni $\mathrm{H}$, et al. Improving Quality Management in Medicine: Is It "Catch 22" All Over Again? Am J Med Qual 2017;7:106286061770026.

15. Leonard M, Graham S, Bonacum D. The human factor: the critical importance of effective teamwork and communication in providing safe care. Qual Saf Health Care 2004;13(Suppl 1):i85-i90.

16. Kobo-Greenhut A, Shnifi A, Tal-Or E, et al. De-freezing frozen patient management. Int J Qual Health Care 2017;29:206-12.

17. Juran JM. Juran's Quality Handbook. 5th ed. New York: McGraw-Hill, 1999.
18. Tatikonda MV, Montoya-Weiss MM. Integrating Operations and Marketing perspectives of product innovation: the influence of organizational process factors and capabilities on development performance. Manage Sci 2001;47:151-72.

19. Hjelmgren D, Dubois A. Organising the interplay between exploitation and exploration: the case of interactive development of an information system. Industrial Marketing Management 2013;42:96-105.

20. Lewis MW, Welsh MA, Dehler GE, et al. Product development tensions: exploring contrasting styles of project management. Acad Manage $J$ 2002;45:546-64.

21. Raisch S, Birkinshaw J, Probst G, et al. Organizational ambidexterity: balancing exploitation and exploration for Sustained Performance. Organization Science 2009;20:685-95. 